\title{
JUANITA, NUEVA VARIEDAD DE NOCHEBUENA PARA INTERIOR DERIVADA POR MUTAGÉNESIS
}

\section{JUANITA, A NEW VARIETY OF POINTSETTIA FOR INDOORS DERIVED BY MUTAGENESIS}

\author{
Jaime Canul-Ku*, Faustino García-Pérez, \\ E. Javier Barrios-Gómez y Sandra E. Rangel-Estrada
}

Instituto Nacional de Investigaciones Forestales, Agrícolas y Pecuarias, Campo Experimental Zacatepec, Zacatepec, Morelos, México.

*Autor para correspondencia (canul.jaime@inifap.gob.mx)

Una de las ventajas del uso de la mutagénesis en materiales élite o variedades en desuso es la posibilidad de cambiar uno o pocos rasgos deseables sin alterar los demás (Hase et al., 2012). Lo anterior, adquiere importancia cuando se irradian con diferentes mutágenos variedades que han permanecido en el mercado por largo tiempo y ya no cumplen con los gustos y preferencias de los consumidores. La aplicación del mutágeno puede conducir a la creación de un material con características diferentes y de interés para el mercado.

En México, existe un mercado potencial de aproximadamente 25 millones de plantas de nochebuena. Para responder a esta demanda el Instituto Nacional de Investigaciones Forestales, Agrícolas y Pecuarias (INIFAP) inició en 2010 el programa de mejoramiento genético de nochebuena (Canul et al., 2017). Las principales estrategias que se han aplicado incluyen la selección, la hibridación y la mutagénesis. El color, la forma de hojas y brácteas son las de mayor interés comercial en nochebuena, además del porte de planta. Éstos se consideran índices útiles en mejoramiento, ya que los cambios ocurridos mediante mutación inducida son fáciles de identificar.

La variedad Juanita se obtuvo mediante mutagénesis recurrente y con selección individual. En 2010, se irradiaron con rayos gamma esquejes sin raíz de una variedad comercial cultivada por productores del estado de Morelos. La irradiación, a una dosis de 5 Gy, se realizó con el equipo Gammacell 220 (Atomic Energy of Canada Limited, Ottawa, Canada) del Instituto de Nacional de Investigaciones Nucleares (ININ), ubicado en Ocoyoacac, Estado de México. Los esquejes irradiados se enraizaron en macetas de tres pulgadas que contenían como sustrato mezcla de ocochal, atocle y polvillo de coco Pelemix $®$ en proporción 60:20:20 v/v/v. Las nuevas plantas se obtuvieron $20 \mathrm{~d}$ después, se cambiaron a macetas de $20.3 \mathrm{~cm}$ de diámetro con el mismo sustrato más lombricomposta de cachaza (principal residuo de la industria de caña de azúcar) en proporción 48:16:16:20 v/v/v/v. En este contenedor se desarrolló la planta hasta la pigmentación de brácteas. El 31 de marzo de 2011 se podaron las plantas y a finales de abril y a inicios del mes de junio se cortaron esquejes, éstos se irradiaron de nuevo a dosis de 5 Gy en el ININ y se enraizaron de la misma forma que en 2010.

En la segunda irradiación se identificaron plantas mutantes, las cuales presentaron hojas con bordes irregulares y de dos colores. En los siguientes ciclos de cultivo, 2012 a 2016, se propagaron vegetativamente las plantas seleccionadas y en éstas se aplicó el método genotécnico de selección individual a las plantas más homogéneas y con el arquetipo definido. La evaluación del material bajo condiciones comerciales se realizó de 2013 a 2016 en Tetela del Monte, Morelos (18 58' latitud norte y $99^{\circ} 15^{\prime}$ longitud oeste). En 2016 se describió la variedad Juanita con base en las directrices de la Unión Internacional para la Protección de las Obtenciones Vegetales (UPOV, 2008).

Juanita se caracteriza por tener porte bajo, de anchura media, con ramificación intermedia. Lo distintivo de este material son sus hojas de forma deltoide con la base de forma cuneiforme y muchos lóbulos de profundidad media. El haz de la hoja muestra dos colores, el principal es verde medio y el secundario verde claro. El color del nervio principal del haz de la hoja es verde y rojo y presenta curvatura de nivel medio. Las brácteas poseen forma oval de color rojo, código 46B en cartas de color (RHS, 2007). El nervio principal de la bráctea no presenta doblamiento ni torsión, pero sí rugosidad intermedia entre los nervios. Las glándulas del ciatio son pequeñas, de color amarillo y no 
se deforman (Figura 1).

Algunas características cuantitativas registradas en Juanita presentan similitud estadística con las del testigo comercial cultivado Prestige, lo cual indica que el tamaño de hoja y bráctea cumplen con los estándares de calidad que demanda el mercado. Uno de los aspectos cualitativos principales es el porte de planta, que junto con la arquitectura conforman la estética de la nochebuena (Cuadro 1).

El Servicio Nacional de Inspección y Certificación de Semillas (SNICS-SADER) otorgó el título de obtentor de Juanita con número de registro 1887. Este material está disponible en el Campo Experimental Zacatepec, Morelos y se puede usar mediante un convenio específico.

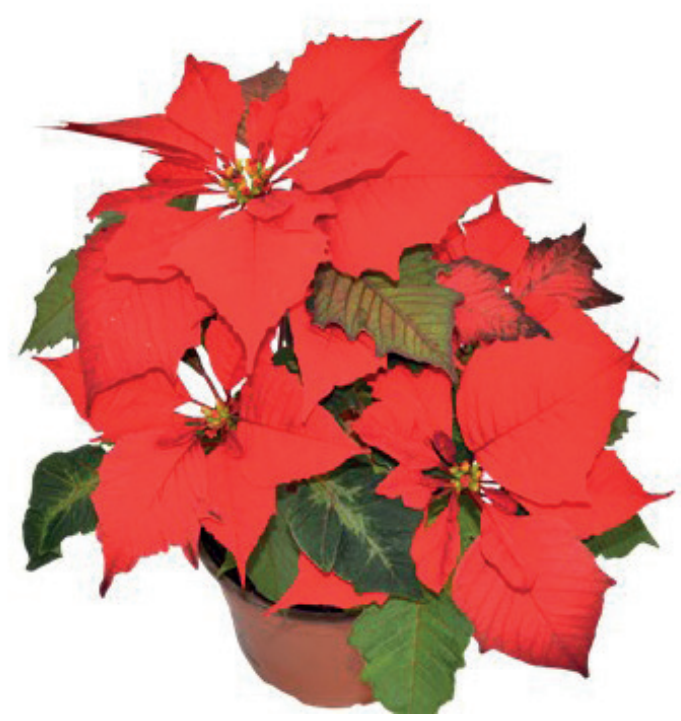

Figura 1. Variedad Juanita en maceta de $20.3 \mathrm{~cm}$ de diámetro.

\section{AGRADECIMIENTOS}

Se agradece el apoyo económico recibido del Instituto Nacional de Investigaciones Forestales, Agrícolas y Pecuarias (INIFAP), el cual permitió la creación de la variedad de nochebuena Juanita.

\section{BIBLIOGRAFÍA}

Canul K. J., F. García P., E. J. Barrios G., S. E. Rangel E., S. G. Ramírez R. y F. J. Osuna C. (2017) Alondra: nuevo híbrido de nochebuena para interiores. Revista Mexicana de Ciencias Agrícolas 8:12031208, https://doi.org/10.29312/remexca.v8i5.119

Hase Y., Y. Akita, S. Kitamura, I. Narumi and A. Tanaka (2012) Development of an efficient mutagenesis technique using ion beams: toward more controlled mutation breeding. Plant Biotechnology 29:193200, https://doi.org/10.5511/plantbiotechnology.12.0106a

RHS, Royal Horticultural Society (2007) R. H. S. Colour Chart. Fifth edition. Royal Horticultural Society. London, England.

UPOV, Unión Internacional para la Protección de las Obtenciones Vegetales (2008) Flor de Pascua, Euphorbia pulcherrima Willd. ex Klotzsch y sus híbridos. Directrices para la Ejecución del Examen de la Distinción, la Homogeneidad y la Estabilidad. TG/24/6 (Proj.3) Ginebra, Suiza. 36 p.

Cuadro 1. Comparación de medias de variables evaluadas en la variedad Juanita y el testigo comercial.

\begin{tabular}{lcccc}
\hline Carácter & Juanita & Testigo comercial & DSH & CV (\%) \\
\hline Altura de planta $(\mathrm{cm})$ & $15.25 \mathrm{a}$ & $14.80 \mathrm{a}$ & 2.05 & 16.11 \\
Longitud de hoja $(\mathrm{cm})$ & $7.23 \mathrm{a}$ & $7.64 \mathrm{a}$ & 0.87 & 13.87 \\
Ancho de hoja $(\mathrm{cm})$ & $4.08 \mathrm{a}$ & $4.19 \mathrm{a}$ & 0.79 & 22.55 \\
Longitud del peciolo de hoja $(\mathrm{cm})$ & $2.55 \mathrm{a}$ & $2.35 \mathrm{a}$ & 0.65 & 31.69 \\
Ancho de bráctea $(\mathrm{cm})$ & $4.58 \mathrm{a}$ & $4.57 \mathrm{a}$ & 0.74 & 19.28 \\
Largo de bráctea $(\mathrm{cm})$ & $8.05 \mathrm{a}$ & $7.96 \mathrm{a}$ & 1.06 & 15.69 \\
Longitud de peciolo de bráctea $(\mathrm{cm})$ & $1.17 \mathrm{a}$ & $1.10 \mathrm{a}$ & 0.17 & 17.63 \\
Amplitud de dosel de bráctea $(\mathrm{cm})$ & $18.23 \mathrm{a}$ & $17.01 \mathrm{a}$ & 2.61 & 17.54 \\
Diámetro de ciatio $(\mathrm{cm})$ & $1.35 \mathrm{a}$ & $1.13 \mathrm{a}$ & 0.26 & 25.33
\end{tabular}

Valores con letras diferentes dentro de hileras indican diferencias significativas (Tukey, 0.05), DSH: diferencia significativa honesta, CV: Coeficiente de variación. 